\title{
Dimensionamento de terraços de infiltração pelo método do balanço volumétrico
}

\author{
Jarbas H. de Miranda ${ }^{1}$, Sergio N. Duarte ${ }^{2}$, Késia O. da Silva ${ }^{3}$ \\ Quirijn de J. van Lier $^{4}$ \& Nilson A. Villa Nova ${ }^{5}$ \\ 1 Departamento de Ciências Exatas - ESALQ/USP. Av. Pádua Dias 11, CP 09, CEP 13418-900, Piracicaba,SP, \\ Fone:(19) 3429-4283. Ramal: 210, E-mail: jhmirand@esalq.usp.br (Foto) \\ 2 Departamento de Engenharia Rural, ESALQ/USP, E-mail: snduarte@esalq.usp.br \\ 3 Instituição Moura Lacerda, E-mail: kosilva@uol.com.br \\ ${ }^{4}$ Departamento de Ciências Exatas, ESALQ/USP, E-mail: qdjvlier@esalq.usp.br \\ ${ }^{5}$ Departamento de Ciências Exatas, ESALQ/USP, E-mail: navnova@esalq.usp.br
}

Protocolo 87 - 9/5/2003 - Aprovado em 1/3/2004

\begin{abstract}
Resumo: As práticas de conservação de solo permitem o controle de perdas de solo e água em áreas agriculturáveis, objetivando assim a maximização do lucro sem provocar a redução da capacidade produtiva. Ante essa importância, há necessidade de se adotar procedimentos técnicos para o dimensionamento de estruturas conservacionistas. Assim, objetivou-se, com o presente trabalho, o desenvolvimento de um modelo computacional em linguagem Visual Basic 6.0, para dimensionamento de terraços em nível. O modelo foi aplicado para 3 localidades do Estado de São Paulo (Araçatuba, Piracicaba e Natividade da Serra). Pelos resultados obtidos, os valores da largura da seção parabólica de canais de terraço em nível de 0,5 m de profundidade para as 3 localidades, com período de retorno de 5 anos, foram de 3,30, 3,34 e 3,80 m, respectivamente.
\end{abstract}

Palavras-chave: conservação de solo e agua, modelagem

\section{Infiltration terrace design by volumetric balance method}

\begin{abstract}
Soil conservation practices allow soil and water loss control in agricultural areas, aiming the maximization of profit without provoking reduction of yield potential. There is need to use technical procedures to design soil conservation structures. Therefore, the aim of the present work was the development of a computational model, in Visual Basic 6.0 language, for design of contour terrace. The model was applied for three municipalities of São Paulo State (Araçatuba, Piracicaba and Natividade da Serra). From the results obtained, it may be concluded that the width of a parabolic section contour terrace channel for these localities, with a return period of 5 years, was $3.30,3.34$ and $3.80 \mathrm{~m}$, respectively, with $0,5 \mathrm{~m}$ of depth.
\end{abstract}

Key words: soil and water conservation, modeling

\section{INTRODUÇÃO}

As práticas conservacionistas permitem o controle de perdas de solo e água em áreas agriculturáveis, objetivando a maximização do lucro sem provocar redução da capacidade produtiva. A erosão consiste no processo de desprendimento e arraste das partículas do solo causado pela ação da água e do vento, constituindo-se na principal causa da degradação de terras agrícolas (Pruski \& Grieseler, 1996). As práticas de conservação do solo podem ser divididas em vegetativas, edáficas e mecânicas. As de caráter vegetativo são aquelas em que se utiliza a vegetação para proteger o solo contra a ação direta das chuvas e, conseqüentemente, minimizar o processo erosivo, enquanto as práticas edáficas são as que, com modificações no sistema de cultivo, além do controle da erosão, mantêm ou melhoram a fertilidade do solo. De acordo com o que se observa na prática, nem sempre as práticas edáficas e vegetativas são suficientes para o controle da erosão, principalmente em regiões em que ocorrem chuvas de grande intensidade. Neste caso, a adoção de procedimentos complementares torna-se necessária para reduzir a velocidade do escoamento superficial e, conseqüentemente, a capacidade de transporte de sedimentos, o que pode ser alcançado mediante a implantação de barreiras mecânicas como, terraços, 
canais escoadouros, bacias de captação de águas pluviais e barragens, entre outros.

O terraceamento em terras agrícolas é uma das práticas mais difundidas entre os agricultores brasileiros para o controle da erosão hídrica, consistindo basicamente na construção de terraços, os quais são estruturas compostas por um dique e um canal, dispostos no sentido transversal à declividade do terreno, formando obstáculos físicos com a intenção de reduzir a velocidade do escoamento superficial e ordenar o movimento da água sobre a superfície do terreno. Segundo Lombardi Neto et al. (1994), a taxa de escoamento superficial é o parâmetro mais importante para dimensionamento de terraços, porém ainda são freqüentes os erros na aplicação desta prática, principalmente no seu dimensionamento correto (Sparovek \& Silva, 1997).

O custo de construção e manutenção de um sistema de terraceamento é relativamente alto; portanto, antes da adoção dessa tecnologia deve-se fazer um estudo criterioso sobre as condições locais, como clima, solo, sistema de cultivo, culturas a serem implantadas, relevo do terreno e equipamento disponível, para que se tenha segurança e eficiência no controle da erosão. O rompimento de um terraço pode levar à destruição dos demais que estiverem a jusante, com grandes prejuízos para a área cultivada (Cruciani, 1987).

O critério corrente para o dimensionamento de terraços em gradiente é a adoção de uma seção capaz de escoar a vazão de escoamento superficial causada por uma chuva de duração igual ao tempo de concentração da área de contribuição; já para os terraços de infiltração ou em nível, dimensiona-se a seção capaz de armazenar o volume de escoamento superficial causado por uma chuva mais longa. Cruciani (1989) sugere que se adote uma chuva de $24 \mathrm{~h}$ com um período de retorno de 5 ou 10 anos. Entretanto, este critério gera seções excessivamente grandes, sobretudo quando o escoamento superficial é calculado pelo método do Número da Curva.

Com o desenvolvimento da ferramenta computacional vêm surgindo modelos capazes de considerar um número maior de fatores intervenientes permitindo realizar o dimensionamento de forma mais precisa. Dentre os modelos utilizados para este fim pode-se citar o modelo matemático "Terraço for Windows", que é um programa computacional desenvolvido por Pruski \& Griebeler (1996), aplicado para o dimensionamento de terraços em gradiente ou em nível utilizando-se, para tal, dados específicos da região e da cultura em questão, como precipitação pluvial máxima esperada para períodos de retorno escolhidos pelo projetista, tipo de solo, taxa de infiltração básica de água no solo, declividade do terreno, manejo do solo, de culturas e de resíduos culturais e altura da crista do terraço, que pode ser construído em função das condições topográficas do terreno e do equipamento disponível para a sua construção.

Entretanto, este programa depende da adoção de determinado período de retorno para a chuva de projeto, obtida por meio da relação intensidade-duração-freqüência da localidade em questão. Como se sabe, muitos estados brasileiros ainda não dispõem dessas relações espacializadas; além disso, o escoamento superficial nem sempre possui o mesmo período de retorno da chuva que o gerou. Assim, considerando-se esses aspectos objetivou-se, com o presente trabalho, o desenvolvimento de um modelo computacional, que pode ser aplicado para avaliação do desempenho de terraços em nível, levando-se em conta os processos de escoamento superficial, infiltração de água no solo e armazenamento de água no terraço.

\section{DESENVOLVIMENTO DO MODELO}

O modelo denominado 'Terradim' foi desenvolvido no Departamento de Ciências Exatas da Escola Superior de Agricultura "Luiz de Queiroz" - ESALQ/USP, em linguagem de programação Visual Basic 6.0 (Fig. 1), O programa opera realizando um balanço volumétrico diário do volume de água armazenado no canal do terraço, durante um período equivalente a uma série de vários anos de dados de precipitação e consiste basicamente de duas etapas: 1. determinação do escoamento superficial diário para uma série longa de dados pluviométricos, mediante a aplicação da metodologia do Número da Curva (Curve Number) e 2. determinação do número de rompimentos da capacidade total de armazenamento de água no canal do terraço.

\section{Determinação do escoamento superficial}

O primeiro procedimento a ser tomado é determinar o valor diário do escoamento superficial, mediante a aplicação do método do Número da Curva $(\mathrm{CN})$.

O modelo permite ao usuário marcar os meses em que será realizado o preparo do solo e, em função disso, interpola a tabela do SCS (USA, 1972) para determinar os valores de CN classes I e III, mediante a Tabela 1.

Tabela 1. Valores do Número da Curva, das classes I, II e III, para os respectivos tipos de cobertura vegetal, solo sem cultivo e solo com cultivo, segundo a precipitação ocorrida nos 5 dias precedentes

\begin{tabular}{ccc}
\hline Classe CN & Solo sem Cultivo & Solo com Cultivo \\
\hline I & $\leq 12,5 \mathrm{~mm}$ & $\leq 35,0 \mathrm{~mm}$ \\
II & 12,5 a $27,5 \mathrm{~mm}$ & 35,0 a $52,5 \mathrm{~mm}$ \\
III & $\geq 27,5 \mathrm{~mm}$ & $\geq 52,5 \mathrm{~mm}$
\end{tabular}

A retenção potencial é função do Número da Curva $(\mathrm{CN})$, cujo valor é tabelado e depende da cobertura do solo, da condição hidrológica, tipo de solo e umidade antecedente do solo. Quanto maior o valor de CN maior também é o escoamento superficial e menor a retenção potencial. Por outro lado, quando se diminui o valor deste parâmetro o escoamento decresce e a retenção aumenta (Eq. 1).

$$
\mathrm{R}=\left(\frac{1000}{\mathrm{CN}_{\mathrm{I}, \mathrm{II}, \mathrm{III}}}-10\right) 25,4
$$

em que:

$\mathrm{R} \quad$ - retenção potencial máxima, $\mathrm{mm}$

$\mathrm{CN}_{\mathrm{I}, \mathrm{II}, \mathrm{III}}$ - número da curva, adimensional

Após o cálculo da retenção potencial máxima, o escoamento superficial é obtido pela Eq. 2. 
A.

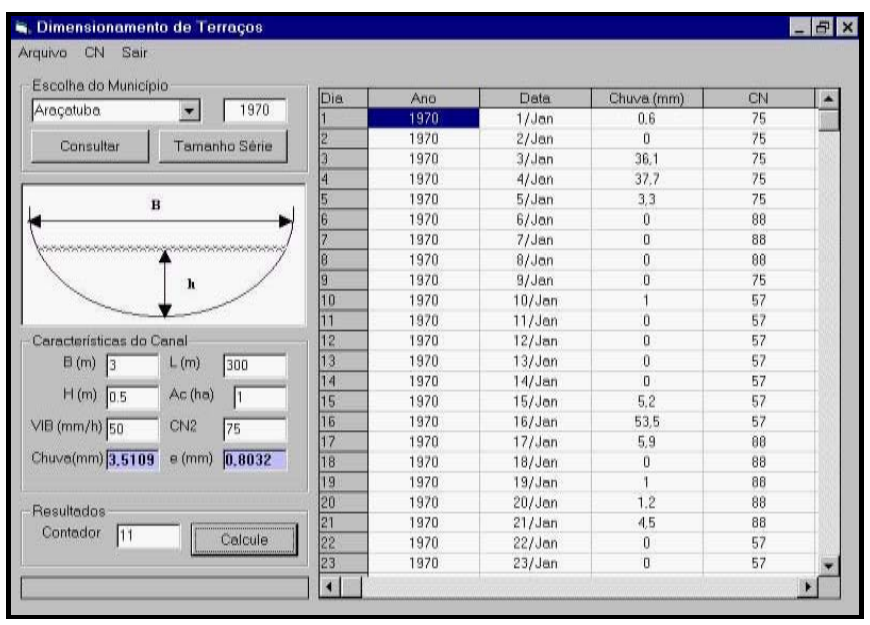

B.

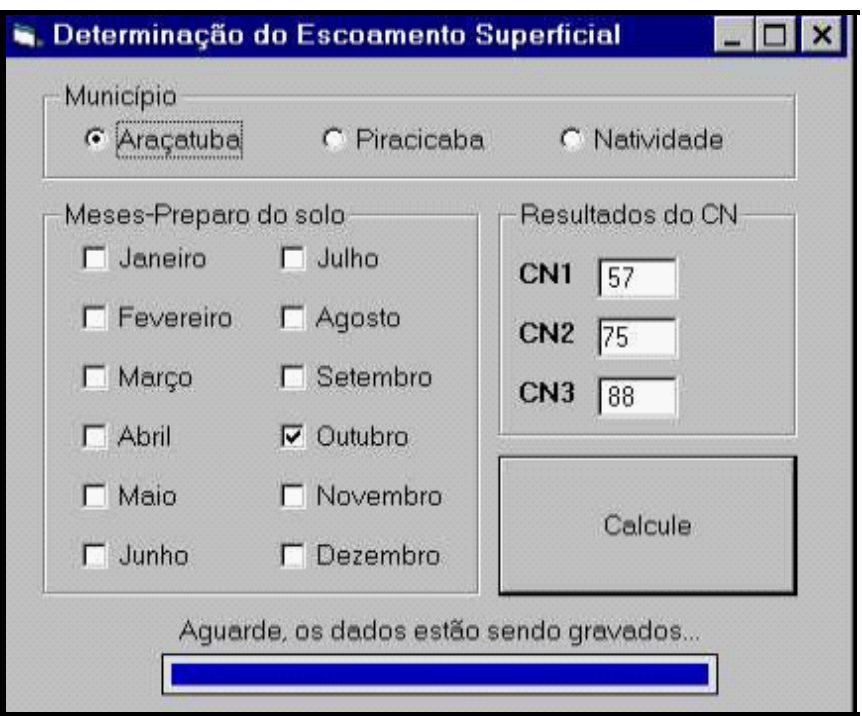

Figura 1. Tela principal do programa Terradim (A) e Tela para o cálculo do escoamento superficial pelo método do Número da Curva (B)

$$
\mathrm{E}_{(\mathrm{i})}=\frac{\left(\mathrm{ppt}_{(\mathrm{i})}-0,2 \mathrm{R}\right)^{2}}{\left(\mathrm{ppt}_{(\mathrm{i})}+0.8 \mathrm{R}\right)}
$$

em que:

$\mathrm{E}_{(\mathrm{i})}$ - escoamento superficial diário, $\mathrm{mm}$

$\mathrm{ppt}_{(\mathrm{i})}$ - precipitação total diária, $\mathrm{mm}$

\section{Estimativa do período de retorno para a seção adotada}

Uma vez obtidos os valores diários de escoamento superficial, o modelo determina o número de rompimentos do terraço durante a série histórica de dados de chuva, ou seja, quantifica o número de vezes que o volume máximo de capacidade de contenção de água pelo terraço foi ultrapassado. Com base neste valor é possível se obter o período de retorno pela relação entre o tamanho da série de dados e o número de rompimentos (Eq. 3). em que:

TR - período de retorno, anos

TS - tamanho da série de dados, anos

NR - número de rompimentos

Para a determinação do número de rompimentos do terraço é conveniente a obtenção de alguns parâmetros, os quais serão abordados a seguir:

2.1. Parâmetros de entrada: primeiro o modelo é alimentado pelos seguintes dados de entrada - área de contribuição do terraço (Ac, ha), largura superior e altura do terraço com seção parabólica $(\mathrm{B}$ e $\mathrm{H}, \mathrm{m})$, valor da velocidade de infiltração básica $\left(\mathrm{VIB}, \mathrm{mm} \mathrm{h}^{-1}\right)$, comprimento do terraço $(\mathrm{L}, \mathrm{m})$ e Número da Curva $(\mathrm{CN})$.

2.2. Determinação da seção do terraço, volume máximo de acumulação e volume diariamente escoado: após o modelo ter sido alimentado pelos parâmetros de entrada, o procedimento na seqüência é a obtenção da seção do terraço, obtida pela Eq. 4:

$$
\mathrm{S}=\frac{2}{3} \mathrm{BH}
$$

em que:

$$
\begin{aligned}
& \mathrm{S} \text { - seção do terraço, } \mathrm{m}^{2} \\
& \mathrm{~B} \text { - base superior do terraço, } \mathrm{m} \\
& \mathrm{H} \text { - altura da lâmina de água no terraço, } \mathrm{m}
\end{aligned}
$$

Uma vez obtida a seção do terraço, o volume máximo de acumulação de água pelo canal é obtido pelo produto entre a seção e o comprimento do terraço (Eq. 5).

$$
\mathrm{V}_{\text {máximo }}=\mathrm{SL}
$$

em que:

$\mathrm{V}_{\text {máximo }}$ - volume máximo de capacidade do terraço, $\mathrm{m}^{3}$

L - comprimento do terraço, $\mathrm{m}$

De posse do valor do escoamento é possível se determinar o volume diariamente escoado, o qual é obtido pelo produto entre o escoamento superficial diário e a respectiva área de contribuição (Eq. 6).

$$
\mathrm{VDE}_{(\mathrm{i})}=\mathrm{E}_{(\mathrm{i})} \mathrm{Ac}
$$

em que:

$\mathrm{VDE}_{(\mathrm{i})}$ - volume diariamente escoado, no dia (i), $\mathrm{m}^{3}$

$\mathrm{E}_{(\mathrm{i})} \quad$ - escoamento superficial, no dia (i), $\mathrm{m}$

Ac - área de contribuição do terraço, $\mathrm{m}^{2}$

2.3. Determinação dos parâmetros geométricos da área molhada do canal do terraço: A seção molhada, base, altura e perímetro molhado foram determinados paras o dias anterior (i-1) e posterior $(i+1)$ pelas seguintes equações:

$$
\mathrm{S}_{(\mathrm{i}-1)}=\frac{\mathrm{VAT}_{(\mathrm{i}-1)}}{\mathrm{L}}
$$


em que:

$\mathrm{S}_{(\mathrm{i}-1)}$ - seção molhada do dia anterior, $\mathrm{m}^{2}$

VAT $_{(\mathrm{i}-1)}$ - volume de água no terraço no dia anterior, $\mathrm{m}^{3}$

$$
\mathrm{S}_{(\mathrm{i}+1)}=\frac{\mathrm{VAT}_{(\mathrm{i}-1)}+\mathrm{VDE}_{(\mathrm{i})}}{\mathrm{L}}
$$

em que:

$\mathrm{S}_{(\mathrm{i}+1)}$ - seção molhada do dia posterior, $\mathrm{m}^{2}$

$\mathrm{VDE}_{(\mathrm{i})}$ - volume diariamente escoado, $\mathrm{m}^{3}$

$$
\mathrm{b}=\left(\frac{3 \mathrm{~s}_{(\mathrm{i}-1)} \mathrm{B}^{2}}{2 \mathrm{H}}\right)^{\frac{1}{3}} \text { ou } \quad \mathrm{b}=\left(\frac{3 \mathrm{~s}_{(\mathrm{i}+1)} \mathrm{B}^{2}}{2 \mathrm{H}}\right)^{\frac{1}{3}}
$$

em que:

b - base superior molhada do terraço, para os dias anterior e posterior, respectivamente, $\mathrm{m}$

A altura de lâmina de água no terraço é obtida pela Eq. 10:

$$
\mathrm{h}=\frac{3}{2} \frac{\mathrm{s}_{(\mathrm{i}-1)}}{\mathrm{b}} \text { ou } \mathrm{h}=\frac{3}{2} \frac{\mathrm{s}_{(\mathrm{i}+1)}}{\mathrm{b}}
$$

em que:

$\mathrm{h}$-altura da lâmina de água no terraço, para os dias anterior e posterior, $\mathrm{m}$

A determinação do perímetro molhado é obtida pela Eq. 11:

$$
\mathrm{P}_{(\mathrm{i}-1)}=\mathrm{b}+\frac{8}{3} \frac{\mathrm{h}^{2}}{\mathrm{~b}} \text { ou } \mathrm{P}_{(\mathrm{i}+1)}=\mathrm{b}+\frac{8}{3} \frac{\mathrm{h}^{2}}{\mathrm{~b}}
$$

em que:

$\mathrm{P}_{(\mathrm{i}-1)}$ - perímetro molhado do dia anterior, $\mathrm{m}$

De posse desses valores determina-se o valor do perímetro molhado médio pela Eq. 12:

$$
\mathrm{P}_{(\mathrm{i})}=\frac{\left(\mathrm{P}_{(\mathrm{i}-1)}+\mathrm{P}_{(\mathrm{i}+1)}\right)}{2}
$$

em que:

$\mathrm{P}_{(\mathrm{i})}$ - perímetro molhado médio, $\mathrm{m}$

Com base no valor do perímetro molhado médio, determinase o valor do volume infiltrado (VI) (Eq. 13):

$$
\mathrm{VI}_{(\mathrm{i})}=\mathrm{VIBP}_{(\mathrm{i})} \mathrm{L}
$$

Conhecendo-se o volume infiltrado pode-se determinar o volume de água no terraço para o dia corrente por meio de um balanço de volumes (Eq. 14).

$$
\mathrm{VAT}_{(\mathrm{i})}=\mathrm{VAT}_{(\mathrm{i}-1)}+\mathrm{VDE}_{(\mathrm{i})}-\mathrm{VI}_{(\mathrm{i})}
$$

em que:

$\mathrm{VAT}_{(\mathrm{i})}$ - volume de água no terraço, $\mathrm{m}^{3}$

Uma vez obtido o valor do volume de água contido pelo terraço, compara-se com o volume máximo de capacidade do terraço e, assim, consegue-se obter quantas vezes ocorreu o rompimento do terraço. De posse do número de vezes em que o terraço foi rompido e no número de anos da série, é possível se estimar o período de retorno para a seção adotada pelo projetista.

As etapas do modelo podem ser conferidas no fluxograma apresentado na Figura 2.

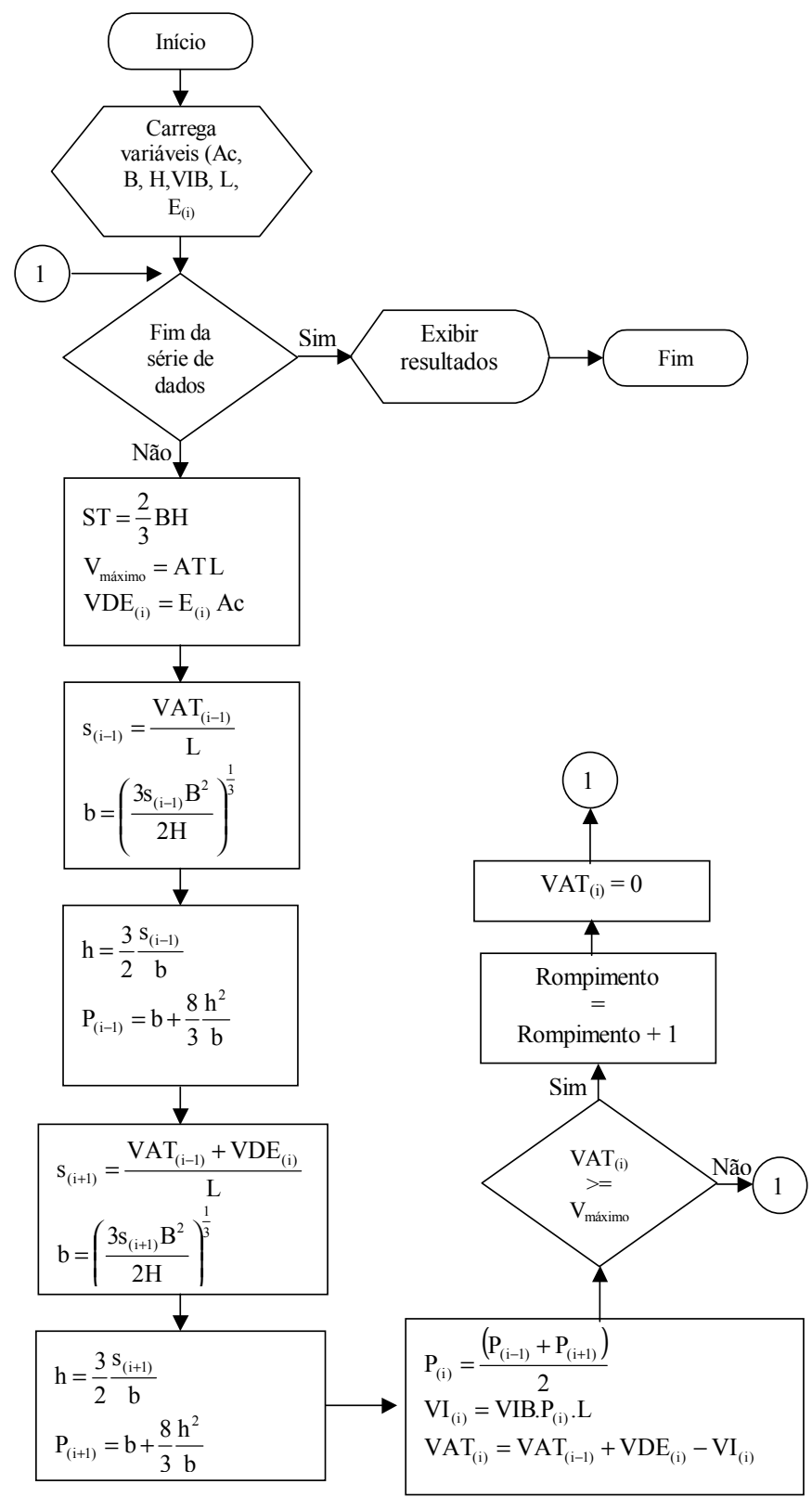

Figura 2. Fluxograma do modelo proposto (Terradim)

\section{APLICAÇÃO DO MODELO}

O presente modelo, “Terradim”, foi aplicado para 3 regiões com comportamentos distintos de chuva no Estado de São 
Paulo. As localidades selecionadas seguiram o critério de ter baixa, média e alta intensidade de chuvas e foram, respectivamente: Araçatuba (Lat.: $21^{\circ} 12^{\prime} \mathrm{S}$, Long.: $50^{\circ} 25^{\prime} \mathrm{W}$ Altitude: $390 \mathrm{~m}$ ), Piracicaba (Lat.: $22^{\circ} 42^{\prime}$ 'S, Long.: 47³8' W e Altitude: $546 \mathrm{~m}$ ) e Natividade da Serra (Lat.: 2323' S, Long.: $45^{\circ} 27^{\prime} \mathrm{W}$ e Altitude: $750 \mathrm{~m}$ ), cujos valores médios anuais de chuva da série de dados de 30 anos (1970 a 1999) foram, respectivamente, $1.281,48 ; 1.344,07$ e $1.570,23 \mathrm{~mm}^{-1}$. Os parâmetros de entrada utilizados nas simulações foram, área de contribuição (Ac), em ha (1 ha), características geométricas do terraço com seção parabólica (B variando de 2 a 5 m, com intervalo de $0,1 \mathrm{~m} \mathrm{e} \mathrm{H}=0,5 \mathrm{~m}$ ), valor da velocidade de infiltração básica $\left(\mathrm{VIB}=50 \mathrm{~mm} \mathrm{~h}^{-1}\right)$, comprimento do terraço $(\mathrm{L}, 300 \mathrm{~m})$ e o Número da Curva $(\mathrm{CN}=75)$.

Para se calcular a duração da chuva que levaria à obtenção das mesmas seções obtidas pelo modelo "Terradim", calculouse o volume do escoamento superficial retido no terraço como o produto da seção pelo comprimento do terraço; obteve-se a lâmina de escoamento dividindo-se o volume pela área de contribuição do terraço; encontrou-se a altura da chuva pelo pelo método do Número da Curva e, finalmente, de forma iterativa, sua duração pelas equações de intensidade-duraçãofreqüência para as localidades de Araçatuba (Eq. 15), Piracicaba (Eq. 16) e Natividade da Serra (Eq. 17), obtidas no programa "Pluvio 1.3" (Pruski \& Silva, 2000).

$$
I=\frac{1660,826 T^{0,134}}{(t+22,456)^{0,795}}
$$

$$
\begin{aligned}
& I=\frac{1773,932 T^{0,173}}{(t+24,999)^{0,798}} \\
& I=\frac{1253,010 T^{0,144}}{(t+18,211)^{0,733}}
\end{aligned}
$$

em que:

I - Intensidade média da chuva, $\mathrm{mm} \mathrm{h}^{-1}$;

$\mathrm{T}$ - período de retorno, anos; e

t - duração da chuva, min.

A Figura 3 apresenta o número de rompimentos do terraço e valores de período de retorno em função dos valores de B (m), para um valor de $\mathrm{H}$ fixado em $0,5 \mathrm{~m}$, para as 3 localidades em questão.

Verifica-se, como esperado, que o número de rompimentos diminui à medida em que se adotam valores de $\mathrm{B}$ maiores. Para Araçatuba, por exemplo, o número de rompimentos variou de 30 a 2, para valores de B variando de 2 a $5 \mathrm{~m}$. A ocorrência de trechos horizontais nos gráficos apresentados para determinada faixa de valores de $\mathrm{B}$, significa que os terraços com essas dimensões apresentam o mesmo número de rompimentos dentro da série testada porém, caso se dispusesse de uma série com maior número de dados, poder-se-ia obter maior refinamento das curvas de número de rompimentos ou período de retorno em função de $\mathrm{B}$.
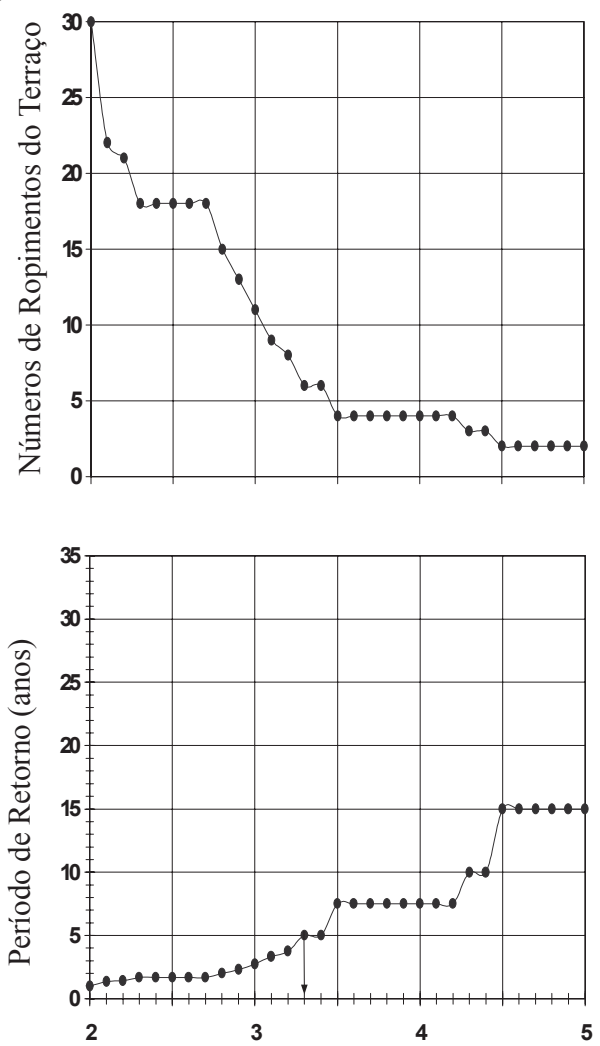

A.
B.
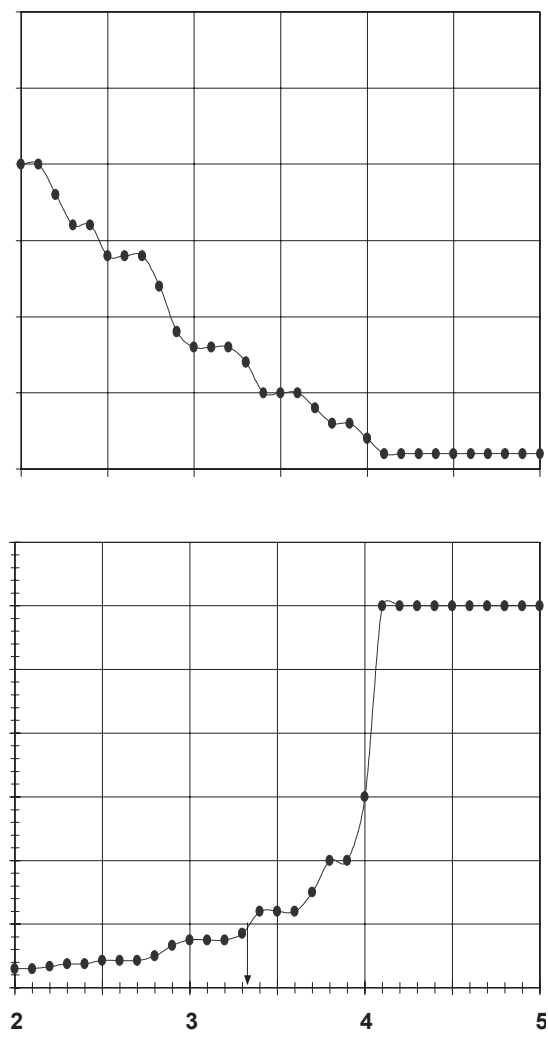

Figura 3. Representação gráfica do número de rompimentos do terraço e valores de período de retorno, em função dos valores da largura (m) superior da seção do terraço para 3 localidades: Araçatuba (A), Piracicaba (B) e Natividade da Serra (C)
C.
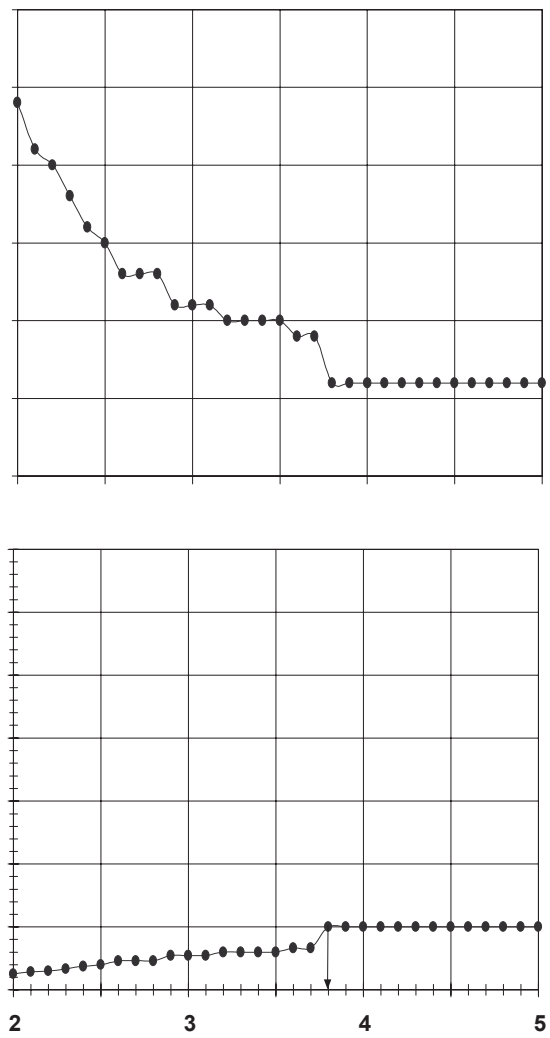
Fixando-se um período de retorno em 5 anos obtém-se, para as localidades de Araçatuba, Piracicaba e Natividade da Serra, valores de $\mathrm{B}$ de 3,30, 3,34 e 3,80 m, respectivamente, cujos resultados estão coerentes, tendo em vista que as localidades apresentam pluviosidade crescente. As seções correspondentes a esses terraços são, respectivamente, 1,10, 1,11 e $1,27 \mathrm{~m}^{2}$, e estão dentro da ordem de grandeza sugerida por Lombardi Neto (1994) que, para terraços em nível, varia de 0,60 a $1,50 \mathrm{~m}^{2}$.

Realizando-se o cálculo inverso, ou seja, verificando-se que chuva levaria à adoção dessas seções, obtiveram-se as alturas de 88,81, 89,23 e 95,89 mm, para as localidades de Araçatuba, Piracicaba e Natividade da Serra, respectivamente. Para um período de retorno 5 anos, segundo as relações intensidade-duração-freqüência, as durações correspondentes são de 2,79, 2,81 e 3,33 h, respectivamente. Verifica-se, portanto, que são valores muito aquém da duração de $24 \mathrm{~h}$ e que sua adoção como critério levaria a superestimativas sensíveis da seção $\left(8,19,9,63\right.$ e 10,90 $\mathrm{m}^{2}$, respectivamente).

\section{CONCLUSÕES}

1. A metodologia de avaliação de desempenho de estruturas hidráulicas por meio da análise de uma longa série de anos, pode também ser aplicada ao dimensionamento de terraços.

2. O modelo forneceu, como resultado, seções de ordem de grandeza coerentes com os dados da literatura e apresentou sensibilidade segundo o regime pluviométrico de cada localidade.

3. O critério da adoção da chuva de $24 \mathrm{~h}$ para o dimensionamento, se aplicado com o método do Número da Curva, geraria seções superdimensionadas.
4. A metodologia proposta permite o dimensionamento de terraços em nível em localidades que não dispõem ainda de relações intensidade-duração-freqüência de chuvas intensas.

\section{LITERATURA CITADA}

Cruciani, D.E. A drenagem na agricultura. 4. ed. São Paulo: Nobel, 1987.337p.

Cruciani, D.E. Dimensionamento de sistemas de drenagem superficial e terraços com base nas características hidrológicas locais. In: Simpósio sobre Terraceamento Agrícola, Campinas : Fundação Cargill, 1989. 266p.

Lombardi Neto, F.; Bellinazzi Júnior, R.; Lepsch, I.F.; Oliveira, J.B. de; Bertolini, D.; Galeti, P.A.; Drugowich, M.I. Terraceamento agrícola. Campinas: Secretaria da Agricultura e do Abastecimento do Estado de São Paulo, Coordenadoria da Assistência Técnica Integral, 1994. 39p. Boletim Técnico, 206

Pruski, F. F. Desenvolvimento de metodologia para o dimensionamento de canais de terraços. Viçosa: Universidade Federal de Viçosa, 1993. 97p. Tese Doutorado

Pruski, F.F.; Griebeler, N.P. Comparação entre métodos para a determinação do volume de escoamento superficial. In: Congresso Brasileiro de Engenharia Agrícola, 25 e Congresso Latino-Americano de Engenharia Agrícola, 2, 1996, Bauru. Anais... Bauru:Sociedade Brasileira de Engenharia Agrícola, 1996. p.422.

Pruski, F.F.; Silva, D.D. Pluvio 1.3: Chuvas intensas para o Brasil. http://www.ufv.br/dea/gprh/pluvio. 2000.

Sparovek, G.; Silva, A. C. Dimensionamento hidrológico de terraços de drenagem e canais escoadouros. Revista da Universidade de Alfenas, Alfenas, v.3, p.137- 143, 1997.

USA. Soil Conservation Service. National engineering handbook: IV. Hydrology. Washington: Department of Agriculture. 1972, n.p. 